\title{
Adolescents and mothers value referral to a specialist service for chronic fatigue syndrome or myalgic encephalopathy (CFS/ME)
}

\author{
Lucy Beasant ${ }^{1}$, Nicola Mills ${ }^{2}$ and Esther Crawley ${ }^{1}$ \\ ${ }^{1}$ School of Social \& Community Medicine, Centre for Child \& Adolescent Health, University of Bristol, \\ Oakfield Grove, UK \\ ${ }^{2}$ School of Social and Community Medicine, University of Bristol, Canynge Hall, 39 Whatley Road, UK
}

\begin{abstract}
Background: Paediatric chronic fatigue syndrome or myalgic encephalopathy (CFS/ME) is relatively common and disabling. Current guidance recommends referral to specialist services, although some general practitioners believe the label of CFS/ME is harmful and many are not confident about diagnosing CFS/ME. Aim: Explore whether or not adolescents and their mothers value referral to a specialist service for young people with CFS/ME. Methods: A qualitative study nested within a feasibility study of interventions for CFS/ME [Specialist Medical Intervention and Lightning Evaluation (SMILE)]. In-depth interviews were undertaken with 13 mothers and 12 adolescents participating in the SMILE study. Transcripts were systematically assigned codes using the qualitative data organisation package NVivo and analysed thematically using techniques of constant comparison. Results: Gaining access to the specialist service was difficult and took a long time. Mothers felt that they needed to be proactive and persistent, partly because of a lack of knowledge in primary and secondary care. Having gained access, mothers felt the CFS/ME service was useful because it recognised and acknowledged their child's condition and opened channels of dialogue between health-care professionals and education providers. Adolescents reported that specialist medical care resulted in better symptom management, although some adolescents did not like the fact that the treatment approach limited activity. Conclusions: Adolescents and their mothers value receiving a diagnosis from a specialist service and making progress in managing $\mathrm{CFS} / \mathrm{ME}$. General practitioners should support adolescents with CFS/ME in accessing CFS/ME specialist services, consistent with current guidance.
\end{abstract}

Key words: adolescents; chronic fatigue syndrome; $\mathrm{ME}$; qualitative; referral; specialist service

Received 5 December 2012; revised 18 February 2013; accepted 9 March 2013;

first published online 25 April 2013

\section{Introduction}

Chronic fatigue syndrome or myalgic encephalopathy $(\mathrm{CFS} / \mathrm{ME})$ is a chronic disabling illness of

Correspondence to: Lucy Beasant, School of Social \& Community Medicine, Centre for Child \& Adolescent Health, University of Bristol, Oakfield House, Oakfield Grove BS8 2BN, UK. Email: Lucy.Beasant@bristol.ac.uk

(C) Cambridge University Press 2013 uncertain aetiology (Royal College of Paediatrics and Child Health, 2004). It is likely to be a complex and multifactorial disorder (Afari and Buchwald, 2003) characterised by debilitating fatigue that is unlike everyday fatigue and can be triggered by minimal activity (NICE, 2007). It is characterised by a range of symptoms including fatigue, malaise, headache, sleep disturbances and difficulty in concentration as well as muscle pain, 
which fluctuate in intensity and severity (NICE, 2007). The estimated prevalence of CFS/ME in young people aged 5-19 years varies between $0.05 \%$ and $2.34 \%$ depending on the definition and diagnostic criteria used (Dobbins et al., 1997; Jordan et al., 2000; Chalder et al., 2003; Farmer et al., 2004; Jones et al., 2004; Rimes et al., 2007; Nijhof et al., 2011). Most GPs will have on average one to three adolescents with CFS/ME on their patient lists (Office for National Statistics, 2011). $\mathrm{CFS} / \mathrm{ME}$ has a negative impact on quality of life and on school attendance, affecting social and emotional development in adolescence (Carter et al., 1999; Bell et al., 2001; van Middendorp et al., 2001; Crawley et al., 2009; Bould et al., 2011). One per cent of secondary school adolescents miss one day a week of school with CFS/ME (Crawley et al., 2011). Guidelines from the National Institute of Health and Clinical Excellence (NICE, 2007) recommend that adolescents with $\mathrm{CFS} / \mathrm{ME}$ are offered access to specialist services immediately if severely affected, within three months if moderately affected and within six months if mildly affected. Despite this, the median time from symptom onset to being seen by a specialist service is still 18 months (Crawley and Sterne, 2009). Literature suggests that there is resistance from primary care practitioners to 'label' an adult with a diagnosis of $\mathrm{CFS} / \mathrm{ME}$, and a more general lack of confidence in labelling clear clinical features of CFS/ME (Bowen et al., 2005). Webb et al. (2011) suggest that GPs would have similar uncertainty when diagnosing and managing adolescents, and concludes that GPs and paediatricians need to be aware of local care pathways and support families accessing NHS services. The aim of this study was to understand the experiences of adolescents and families in accessing and using a specialist service, and explore whether or not they value referral to a specialist service for young people with $\mathrm{CFS} / \mathrm{ME}$.

\section{Methods}

\section{Study group}

We recruited participants from the Specialist Medical Intervention and Lightning Evaluation (SMILE) study designed to test the feasibility and acceptability of recruiting adolescents to a randomised controlled trial (RCT) comparing specialist medical care with specialist medical care and the
Lightning Process. Further information about the SMILE study can be found at: http://www.bristol. ac.uk/ccah/research/childrencomplexhealthneeds/ chronic-fatigue/smile.html. Adolescents referred to a large regional specialist CFS/ME service in South West England were assessed by specialist clinicians at their first appointment for study eligibility. Participants were eligible for the SMILE study if they had been diagnosed with CFS/ME, were aged between 12 and 18 years and were mildly or moderately affected by the condition; that is, they were not house bound (NICE, 2007). Clinicians informed eligible participants about the SMILE study and interested participants were visited at home a few days later by a research officer to discuss the study in more detail and to be randomised to either intervention if willing. In-depth qualitative interviews were undertaken with adolescents and their mothers who had agreed to participate in the SMILE study to determine their views on the specialist service, study participation and the interventions received.

\section{Sampling, data collection and analysis}

We purposively sampled those participating in the SMILE study to ensure that interviews included a range of participants in terms of age, sex, socioeconomic circumstance and ethnicity as well as families from both intervention arms (maximum variation sampling), with the potential to target participants with characteristics of interest to follow-up and develop emerging findings (theoretical sampling). Families were interviewed at three possible time points: after initial assessment at the specialist clinic and before randomisation, after randomisation but before the intervention, and after the intervention. Adolescents with CFS/ME were interviewed once at one of these time points for not more than $20 \mathrm{~min}$.

Parent interviews lasted for $20-60 \mathrm{~min}$ and were conducted at a convenient location, usually at the participants' homes. A checklist of topics was used to ensure that similar areas were covered in each interview but with sufficient flexibility to enable participants to raise topics of interest to them. The guide covered questions concerning experiences of the initial clinical assessment appointment, study participation and the interventions that young people received (see Appendix for checklist of topics). 
Table 1 Comparison of characteristics of adolescents aged 12-18 years diagnosed with CFS/ME by the paediatric CFS/ME service between $01 / 09 / 2010$ and 31/12/2011, with the sample of adolescents interviewed for qualitative analysis

\begin{tabular}{lccc}
\hline & Interviewed $(n=12)$ & CFS cohort $(n=129)$ & P-value $^{\text {a }}$ \\
\hline Female (\%) & $9(75.0 \%)$ & $103(79.8 \%)$ & 0.71 \\
Age (years), mean (SD) & $13.9(1.6)$ & $14.8(1.6)$ & 0.07 \\
Illness duration, median (IOR) & $13(9$ to 18$)$ & $12(8$ to 24$)$ & 0.99 \\
\hline
\end{tabular}

$\mathrm{CFS} / \mathrm{ME}=$ chronic fatigue syndrome/Myalgic Encephalopathy.

${ }^{a}$ Fisher's exact test for comparison of proportions, Student's $t$-test for comparison of mean values, Mann-Whitney two-sample test for comparison of medians.

Interviews were audio-recorded and transcribed verbatim. Data items were systematically assigned codes using the qualitative data organisation package NVivo (QSR International Pty Ltd, 2010) and analysed thematically using techniques of constant comparison (Glaser and Strauss, 1967). The constant comparative approach enabled early data analysis, and this led to the addition of two prompts on the interview schedule. One prompt initiated discussion about 'how families got to the CFS/ME service' if mothers had not already discussed this, but the majority of mothers initiated discussion about this topic. Perspectives of participants were of paramount importance, with careful account taken of the context within which the discussion took place. Data analysis was an ongoing and iterative process, commencing soon after data collection started and informing further sampling and data collection. Two members of the research team analysed $\sim 10 \%$ of the data independently to compare coding and enhance its reliability. Descriptive accounts were produced, and theoretical explanations for behaviours, opinions and decisions were developed.

\section{Results}

\section{Participants}

Thirteen mothers were approached and all were interviewed. Five mothers were interviewed at all three time points to form case studies. The remaining eight mothers took part in one-off interviews: four post randomisation and four after their child received an intervention. Fifteen adolescents were approached to be interviewed. One mother did not consent to her child being interviewed, and two adolescents (a 16-year-old boy and a 14-year-old girl) declined. Of the 12 adolescents, five were interviewed post randomisation but before receiving the intervention, and seven after the intervention. Three adolescents were male and nine were female.

Table 1 shows baseline characteristics of the adolescents interviewed and those seen by the service during the same time period. The mean age of interviewed adolescents was 13.9 years; $75 \%$ were female and the median illness duration was 13 months. Those interviewed were similar to those who were diagnosed at the specialist clinic, except that they were slightly younger (mean age 13.9 compared with 14.8 years, $P=0.07$ ).

\section{Thematic analysis}

Thematic analysis was performed using quotes about specialist medical care from mothers and adolescents. All mothers and adolescents accessed specialist medical care and all commented on the care received. All mothers and some adolescents commented on their journey before the assessment.

Three themes were identified around parent and adolescent experiences of accessing a specialist service:

1. Long and difficult journey to the CFS/ME service.

2. Recognition and progress, taking the next steps.

3. Dialogue opened between health-care professionals and education providers.

\section{Long and difficult journey to the \\ CFS/ME service}

Nearly all mothers reported a period of prolonged illness, and most described a long and difficult journey to the CFS/ME service. Mothers felt that there was a lack of initial guidance or information around CFS/ME and day-to-day 
management of symptoms before accessing the specialist service, leading to initial confusion and uncertainty about their child's health and possible diagnosis of $\mathrm{CFS} / \mathrm{ME}$ :

'I feel as though it's in the later stages, I can see the light at the end of the tunnel now, I couldn't see that 2 years ago... we weren't even given a form [giving information about $\mathrm{CFS} / \mathrm{ME}$ ] when [young person, YP8] was first diagnosed. Not even a piece of paper. It was disgusting'

(Mother of YP8: female aged 16 years)

'because at the beginning you're just going out of your mind because you don't know what's wrong with your child, and because extreme tiredness can be a symptom of any number of different illnesses, you really feel completely at sea'

(Mother of YP2: female aged 14 years)

Before accessing the specialist CFS/ME service, there was also confusion around diagnosis, which was either uncertain or given as CFS/ME but with a lack of conviction:

'[paediatrician said] we'll see if all the blood tests are clear, which they were, so they said yeah, you've definitely, probably got ME'

(Mother of YP7: male aged 13 years)

'at the [hospital] they did lumbar punctures and a brain scan just to rule out anything nasty and it was actually myself that suggested to them that perhaps it was an ME type of condition'

(Mother of YP12: female aged 15 years)

The journey to the CFS/ME service was deemed complex and frustrating with numerous interactions with health-care professionals at various locations, and long periods of waiting intensified by repeated blood tests to rule out serious acute illnesses:

'It took ages, it took absolutely ages... she had lots and lots of blood tests and things, and then we had to wait for them to come back, then she had to have some more tests ...its good they've eliminated some, some nasty illnesses but then you're no further forward in understanding what it's all about'

(Mother of YP2: female aged 14 years)
A small number of mothers reported having to wait for funding to be agreed before their child could access the specialist CFS/ME service.

Co-morbid conditions further complicated the journey to the $\mathrm{CFS} / \mathrm{ME}$ service by introducing complexity to the process of diagnosis or masking $\mathrm{CFS} / \mathrm{ME}$ symptoms and hindering symptom management. Nearly all mothers suggested that other conditions, such as behavioural issues or depression, had developed because of prolonged illness with $\mathrm{CFS} / \mathrm{ME}$ :

'looking back YP12 had like awful behaviour which was really upsetting as a parent, really aggressive and nasty, which she was at times, which I think was all to do with her illness'

(Mother of YP12: female aged 15 years)

Mothers felt that they had to be proactive and persistent, using additional knowledge sources to bypass potential gatekeepers who acted as a barrier because of a lack of knowledge about either $\mathrm{CFS} / \mathrm{ME}$, potential treatment or availability of specialist services. This was felt to be the case for both GPs and paediatricians:

'I had to go back to the GP and say could you refer us to this clinic that's being organised? and he said "I'm quite happy to refer you, but I have to say it doesn't help everybody"... and I was cross because I thought, so you knew about this clinic and he hadn't said "Right, this is what we'll do" ... we were not referred straight away and I think that comes back to the GPs; I think at GP level there's still either ignorance or doubt'

(Mother of YP34: male aged 15 years)

'I asked [paediatrician] could she be seen by somebody from the CFS/ME service because I've heard they do a lot more research and he said to me, that "they do nothing that I don't do"... that was his exact words... and it was then that we decided, right, no YP8 needs to see somebody else'

(Mother of YP8: female aged 16 years)

Recognition and progress - taking the next steps

The majority of mothers reported the initial assessment appointment with the CFS/ME service as a positive experience, which was useful and helpful. The service recognised and acknowledged

Primary Health Care Research \& Development 2014; 15: 134-142 
the young person's condition, resulting in a sense of relief and reassurance. Mothers felt that symptoms were now being understood and they would receive help:

'and it was a big, such a relief when he got there [CFS/ME service] ...just to sort of have someone saying "Yes, this is what you've got" and this is what you can do'

(Mother of YP34: male aged 15 years)

Referral to a specialist service gave families access to an informative team of experts, for some a formal diagnosis, and for all a tailored, patientcentred specialist medical intervention that had not been available earlier. This enabled positive change and steps towards a managed recovery:

'it was absolutely amazing, she [CFS/ME service clinician] knew exactly what she was talking about and I didn't feel that [paediatrician] did....in fact I found with a lot of the doctors that we've seen, to be honest with you, not all of them accept it [CFS/ME]'

(Mother of YP8: female aged 16 years)

'I think it was really empowering for us to feel we had a form to follow, we had a technique to apply and that really, really helped'

(Mother of YP36: female aged 13 years)

'I found this concrete information that we've got from our appointment much, much better'

(Mother of YP4: male aged 12 years)

Some mothers felt that the CFS/ME service reinforced symptom management strategies that they had been trying to get their child to follow, and that they felt their child would be more likely to listen if techniques were legitimised by a health-care professional:

'although she does listen to her mum, they don't at her age it's better coming from a professional, or better to be reinforced by a professional, because it just comes across as mum nagging'

(Mother of YP88: female aged 17 years)

Half the adolescents reported that specialist medical care was positive, as it enabled them to talk about their illness (as opposed to continuing with investigative tests and procedures) and gave guidance on how to manage their condition. This brought structure and a sense of normality back into their lives:

'I thought they [CFS/ME service] were quite helpful; I think just to talk about things it is really good'

\section{(YP12: female aged 15 years)}

'I think it's [specialist medical care] really good and it's really useful and helpful, and you know what you can do ... rather than what you can't do...so it's quite good to know that you can still do like normal stuff, just not so much of it and it's really good'

(YP2: female aged 14 years)

However, half reported that, although specialist medical care resulted in better symptom management, accepting that for a time they must reduce activity levels and adopt a routine was challenging:

'YP34 didn't take very kindly to the initial suggestions of their course of treatment ... what he really resented was the sort of the limited, going to bed, strict routine, getting up and things'

(Mother of YP34: male aged 15 years)

A few mothers also noted that specialist medical care strategies had an impact on the whole family and could be difficult to integrate with their routine lifestyle:

'The appointments are one thing, but going home and implementing these things had a huge impact on our lifestyle at home'

(Mother of YP36: female aged 13 years)

\section{Dialogue opened between health-care \\ professionals and education providers}

Mothers discussed the beneficial way in which the CFS/ME service opened channels of dialogue between health-care professionals and education providers in a variety of ways. A letter provided by the CFS/ME service confirming a diagnosis enabled mothers to legitimately take their child out of school, request funding for home schooling and more generally inform and gain support from teachers when managing reduced attendance:

'the teachers at that point hadn't been very supportive...they said YP12 was faking, that 
wasn't the word used but you know it was the body language and things like that you could see that "oh another teenager trying it on sort of thing", so it was really nice to get support...they did that for me, they actually wrote to the school and explained'

(Mother of YP12: female aged 15 years)

\section{Discussion}

This study showed that, despite the largely positive experience of using a specialist service, the journey to it was long and difficult, dependent partially on whether professionals knew about the service and treatment available. The specialist service provided a firm diagnosis and treatment plan, recognition of what was happening and improved support from education providers; however, some adolescents did not like the fact that the treatment approach limited activity.

\section{Strengths and weaknesses of the study}

This is the first study to investigate both adolescent and parent views on specialist CFS/ME services. By using in-depth interviews, participants were able to convey complex accounts and discuss issues that they felt to be important, providing a rich data set that may not have emerged through more structured questionnaire-based research. Parent/adolescent relations during a chronic illness can be complex, with adolescents challenging their parents as authority figures. Interviewing mothers and adolescents enabled us to capture some of this complexity.

The specialist paediatric CFS/ME service is a well-established service in an area that covers rural and urban populations. Although the service accepts referrals from general practitioners, it also accepts referrals from secondary care. Patients accessing the specialist service may be more severely affected or their condition may be more complex than those seen in primary and secondary care, and these results may not be transferable to community or secondary care services.

Those recruited to this study were part of a feasibility RCT. Patients recruited to RCTs can differ from those who are eligible but not recruited to a trial (Rothwell, 2005), and therefore these results may not be transferable to non-trial participants. Adolescents who participated in the SMILE study were mild-to-moderately affected by $\mathrm{CFS} / \mathrm{ME}$, and therefore the experiences of young people with severe $\mathrm{CFS} / \mathrm{ME}$ and their families might be different. However, the adolescents who participated in this study were similar to the larger clinic population, and characteristics of informants have been given so that readers can judge transferability of findings to their own patient group.

In this study, only mothers consented to be interviewed. This may be because mothers were more likely to attend the initial assessment appointment with their child (Missen et al., 2011) or because mothers still take on more 'caring' responsibilities within the family, and either take time off from work or work part time to attend clinical or interview appointments.

\section{Comparison with existing literature}

Mothers had difficulty accessing the specialist service, which they felt was because of lack of knowledge from both GPs and paediatricians about diagnosis or available treatment. This is consistent with a report from 'Action for ME' (2008) where one in three adults and young people said that their GP was either unsupportive or less informed than they should be about CFS/ME. Only $52 \%$ of GPs feel confident diagnosing CFS/ME in adults (Bowen et al., 2005), and GPs may be less confident in diagnosing adolescents. In addition, many GPs feel that the label of $\mathrm{CFS} / \mathrm{ME}$ can be harmful because it does not offer a clear management pathway for patients, although other GPs believe that the labelling effect is limited and short-lived (Chew-Graham et al., 2010).

There is considerable debate about the value of labels in adult fatigue syndromes (Hamilton et al., 2005; Huibers and Wessely, 2006), with some suggesting that using the label CFS/ME may lead to pessimistic beliefs and a self-fulfilling prophecy, triggering or validating perceptions of ill health and perpetuating and exaggerating symptoms (Huibers and Wessely, 2006). However, our study suggests that, for adolescents, a label can be helpful by shifting the illness from unknown to known and stopping the repetition of ambiguous tests and procedures, which can contribute to an overall 'difficult emotional experience' for adolescents with CFS/ME (Jelbert et al., 2010). Confirmation by the medical profession and a 
belief in mothers' observations of their child's ill health also legitimises the struggle mothers have experienced in pursuing access to services.

Mothers and young people in this study valued the specialist service because it provided a way forward in terms of treatment and improved dialogue between professionals. NICE guidance 2007 makes clear recommendations for the diagnoses and treatment of CFS/ME, including referral to specialist services. Results of an ME Association Survey (The ME Association, 2010) suggested that patients and carers rated 'a specialist CFS/ME centre' as their first choice when asked where they would like to be treated. However, there are few services for adolescents with CFS/ME, as highlighted by the all-party inquiry into NHS service provision (2010), which suggested that this had led to many not receiving adequate care.

\section{Implications for practice and research}

Adolescents and their mothers value receiving a diagnosis from a specialist CFS/ME service and making progress in managing their condition, but access to such a service is a long and difficult process. Primary care services should better support adolescents with CFS/ME in gaining access to specialist health care and professional advice, consistent with current NICE guidelines (NICE, 2007). Further research is needed to investigate whether labelling in paediatric CFS/ME changes outcome or engagement in specialist medical care.

\section{Acknowledgements}

The authors thank the participants who shared their time and experiences.

\section{Funding}

This study was supported by The Lindbury Trust and The Ashden Trust. This work is produced by Dr Esther Crawley under the terms of a Clinician Scientist Award issued by the National Institute for Health Research. Dr Nicola Mills is funded by the Bristol ConDuCT Hub of the MRC Hubs for Trials Methodology Research. The views expressed in this publication are those of the author(s) and not necessarily those of the NHS, the National Institute for Health Research, MRC or the Department of Health.

\section{Ethical approval}

South West 2 Local Research Ethics Committee reference 10/H0206/32.

\section{Conflict of interest}

Dr Crawley is a medical advisor for the Association for Young people with ME, (AYME) and the Sussex and Kent ME society.

\section{References}

Action for ME. 2008: What progress? Initial findings of a national survey of over 2,760 people with M.E. focusing on their health and welfare. Published by Action for M.E. and AYME for M.E. Awareness Week, May 2008.

Afari, N. and Buchwald, D. 2003: Chronic fatigue syndrome: a review. American Journal of Psychiatry 160, 221-36.

All-Party Parliamentary Group on ME. 2010: Inquiry into NHS service provision for ME/CFS.

Bell, D.S., Jordan, K. and Robinson, M. 2001: Thirteen-year follow-up of children and adolescents with chronic fatigue syndrome. Pediatrics 107, 994-98.

Bould, H., Lewis, G., Emond, A. and Crawley, E. 2011: Depression and anxiety in children with CFS/ME: cause or effect? Archives of Disease in Childhood 96, 211-14.

Bowen, J., Pheby, D., Charlett, A. and McNulty, C. 2005: Chronic Fatigue Syndrome: a survey of GPs' attitudes and knowledge. Family Practice 22, 389-93.

Carter, B.D., Kronenberger, W.G., Edwards, J.F., Marshall, G.S., Schikler, K.N. and Causey, D.L. 1999: Psychological symptoms in chronic fatigue and juvenile rheumatoid arthritis. Pediatrics 103, 975-79.

Chalder, T., Goodman, R., Wessely, S., Hotopf, M. and Meltzer, H. 2003: Epidemiology of chronic fatigue syndrome and self reported myalgic encephalomyelitis in 5-15 year olds: cross sectional study. BMJ 327, 654-55.

Chew-Graham, C., Dowrick, C., Wearden, A., Richardson, V. and Peters, S. 2010: Making the diagnosis of Chronic Fatigue Syndrome/Myalgic Encephalitis in primary care: a qualitative study. BMC Family Practice 11, 16.

Crawley, E. and Sterne, J.A. 2009: Association between school absence and physical function in paediatric chronic fatigue syndrome/myalgic encephalopathy. Archives of Disease in Childhood 94, 752-56.

Crawley, E., Hunt, L. and Stallard, P. 2009: Anxiety in children with CFS/ME. Eurpean Child \& Adolescent Psychiatry 18, 683-89.

Crawley, E., Emond, A.M. and Sterne, J.A. 2011: Unidentified Chronic Fatigue Syndrome/myalgic encephalomyelitis (CFS/ME) is a major cause of school absence: surveillance outcomes from school-based clinics. BMJ Open 1, e000252.

Dobbins, J.G., Randall, B., Reyes, M., Steele, L., Livens, E.A. and Reeves, W.C. 1997: The prevalence of chronic fatiguing 
illnesses among adolescents in the United States. Journal of Chronic Fatigue Syndrome 3, 15-27.

Farmer, A., Fowler, T., Scourfield, J. and Thapar, A. 2004: Prevalence of chronic disabling fatigue in children and adolescents. British Journal of Psychiatry 184, 477-81.

Glaser, B.G. and Strauss, A.L. 1967: The discovery of grounded theory. USA: Aldine Transaction.

Hamilton, W.T., Gallagher, A.M., Thomas, J.M. and White, P.D. 2005: The prognosis of different fatigue diagnostic labels: a longitudinal survey. Family Practice 22, 383-88.

Huibers, M.J. and Wessely, S. 2006: The act of diagnosis: pros and cons of labelling chronic fatigue syndrome. Psychological Medicine 36, 895-900.

Jelbert, R., Stedmon, J. and Stephens, A. 2010: A qualitative exploration of adolescents' experiences of chronic fatigue syndrome. Clinical Child Psychology and Psychiatry 15, 267-83.

Jones, J.F., Nisenbaum, R., Solomon, L., Reyes, M. and Reeves, W.C. 2004: Chronic fatigue syndrome and other fatiguing illnesses in adolescents: a population-based study. Journal of Adolescent Health 35, 34-40.

Jordan, K.M., Ayers, P.M., Jahn, S.C., Taylor, K.K., Huang, C.F., Richman, J. and Jason, L.A. 2000: Prevalence of fatigue and chronic fatigue syndrome-like illness in children and adolescents. Journal of Chronic Fatigue Syndrome 6, 3-21.

Missen, A., Hollingworth, W., Eaton, N. and Crawley, E. 2011: The financial and psychological impacts on mothers of children with chronic fatigue syndrome (CFS/ME). Child: Care, Health and Development 38, 505-12.

National Institute for Health and Clinical Excellence. 2007: Chronic fatigue syndrome/Myalgic encephalomyelitis (or encephalopathy); diagnosis and management. CG53. London: NICE.

Nijhof, S.L., Maijer, K., Bleijenberg, G., Uiterwaal, C.S., Kimpen, J.L. and van de Putte, E.M. 2011: Adolescent chronic fatigue syndrome: prevalence, incidence, and morbidity. Pediatrics 127, e1169-75.

NVivo qualitative data analysis software; QSR International Pty Ltd. Version 9. 2010.

Office for National Statistics. 2011: Population estimates for UK, England and Wales, Scotland and Northern Ireland, mid-2010. Retrieved 21 December 2011 from http://www. ons.gov.uk/ons/publications/re-reference-tables.html?edition= tcm \%3A77-231847

Rimes, K.A., Goodman, R., Hotopf, M., Wessely, S., Meltzer, H. and Chalder, T. 2007: Incidence, prognosis, and risk factors for fatigue and chronic fatigue syndrome in adolescents: a prospective community study. Pediatrics 119, e603-09.

Rothwell, P.M. 2005: External validity of randomised controlled trials: "to whom do the results of this trial apply?". Lancet 365, 82-93.

Royal College of Paediatrics and Child Health. 2004: Evidence based guideline for the management of CFS/ME (chronic fatigue syndrome/myalgic encephalopathy) in children and young people. London: Royal College of Paediatrics and Child Health.
The ME Association. 2010: Managing my M.E. Survey of illness management requirements. The ME Association.

van Middendorp, H., Geenen, R., Kuis, W., Heijnen, C.J. and Sinnema, G. 2001: Psychological adjustment of adolescent girls with chronic fatigue syndrome. Pediatrics 107, E35.

Webb, C.M., Collin, S.M., Deave, T., Haig-Ferguson, A., Spatz, A. and Crawley, E. 2011: What stops children with a chronic illness accessing health care: a mixed methods study in children with Chronic Fatigue Syndrome/Myalgic Encephalomyelitis (CFS/ME). BMC Health Services Research 11, 308.

\section{Appendix}

\section{Interview Topic Guide \\ *Questions used to elicit responses to accessing a specialist CFS/ME service.}

These questions will be used as prompts to ensure all important areas are covered.

Welcome, introduction, discuss confidentiality \& consent, sign consent form. Check continues to be happy with consent process.

\section{After assessment \& before randomisation}

1. *Can you talk me through YP's initial appointment with the specialist?

Prompts: What was said, did you understand what was being said? Feelings?

Additional prompt: How did [child] get to the $\mathrm{CFS} / \mathrm{ME}$ service?

2. *What were your initial thoughts about the study?

Prompts: What did you think when you were told about it? Feelings? Worries? Expectations?

3. What did you think about the information you were given about the study?

Prompts: What information did you get - oral and written (PIS)? Did you read it? Understand it? Did it give you enough information/too much? Were there things you thought they had forgotten to include?

4. Did you know anything about the Lightning Process before this initial appointment (for first interview only)?

Prompts: How/ who? What did you think? What information? 
5. Have you found out any information about the Lightning Process since?

Prompts: Why? How? What did you find? What did you think?

6. What are your thoughts at this stage on taking part or not? Why?

NB - Stress that they're not being asked at this stage but that we want to gauge their thoughts, stress also that it makes no difference to the interviewer

7. *If you were to take part, would you have a preference for one of the interventions?

Prompts: Why? What would you do if allocated the other intervention? Issues over participation? Engagement?

8. What do you think about having treatments allocated at random, i.e. by chance?

Prompts Why is it done? How do you feel about this way of deciding what treatment you'll get? Is there a better way? Do you think you'll be happy to be randomised? Do you think you're likely to get one intervention rather than the other? Why?

9. *You have now done some questionnaires at follow up. What did you/your child think about the questions you were asked?

Prompts: Were there any particularly difficult ones? What did you think about the HADS/ POMS inventory? Would you leave some out? Other areas that should be covered?

\section{After randomisation \& before interventions}

1. Can you tell me what happened when the research nurse visited and explained about randomisation?

Prompts: What did she say? Understandable? What did you think? Did you understand what was going to happen?

2. What did you think before randomisation?

Prompts: Were you happy with the process? Did you understand what was going to happen and why?
3. Did you agree to randomisation or not? Why?

4. *What did you think when you got your intervention allocation?

Prompts: How did you feel? Was it what you expected/wanted? Expectations of intervention? What have you done since then?

5. You have now done some questionnaires at follow up. What did you/your child think about the questions you were asked?

Prompts: Were there any particularly difficult ones? What did you think about HADS/ POMS inventory? Would you leave some out? Other areas that should be covered?

\section{After intervention}

1. *Tell me about the intervention you received? Prompts: What happened? What was good/bad? What would you change? Venue? Structure of sessions? Language used? Was it as expected?

2. Do you think you/your child have/has learnt anything from it, if so what?

Prompts: About CFS/ME, themselves, self management?

3. What has happened after the intervention?

Prompts: How have you/they done? What are you/they doing? Feeling?

Additional prompt: How did LP fit with SMC? How did you manage conflict [if reported by participant]

4. What do you think now about being randomised? Prompts: Would you do it again? What do you think about the study for others?

5. You have now done some questionnaires at follow up. What did you/your child think about the questions you were asked?

Prompts: Were there any particularly any difficult ones? What did you think about the HADS/ POMS inventory? Would you leave some out? Other areas that should be covered? 